\title{
Transition from drift to interchange instabilities in an open magnetic field line configuration
}

\author{
F. M. Poli, ${ }^{a}{ }^{2}$ P. Ricci, A. Fasoli, and M. Podestà ${ }^{\text {) }}$ \\ CRPP-EPFL, Association EURATOM-Confédération Suisse, Lausanne, Switzerland
}

(Received 10 December 2007; accepted 25 February 2008; published online 26 March 2008)

\begin{abstract}
The transition from a regime dominated by drift instabilities to a regime dominated by pure interchange instabilities is investigated and characterized in the simple magnetized toroidal device TORPEX [TORoidal Plasma EXperiment, A. Fasoli et al., Phys. of Plasmas 13, 055906 (2006)]. The magnetic field lines are helical, with a dominant toroidal component and a smaller vertical component. Instabilities with a drift character are observed in the favorable curvature region, on the high field side with respect to the maximum of the background density profile. For a limited range of values of the vertical field they coexist with interchange instabilities in the unfavorable curvature region, on the plasma low field side. With increasing vertical magnetic field magnitude, a gradual transition between the two regimes is observed on the low field side, controlled by the value of the field line connection length. The observed transition follows the predictions of a two-fluid linear model. (C) 2008 American Institute of Physics. [DOI: 10.1063/1.2899303]
\end{abstract}

\section{INTRODUCTION}

Among the modes driving turbulence in the scrape-off layer (SOL) of fusion devices, drift waves and interchange instabilities play a major role. Drift waves arise in the presence of plasma density gradients and can be driven unstable by resistivity, finite electron mass, and Landau damping. Their growth rate is $\gamma \sim \omega_{*}$, where $\omega_{*}=k_{\perp} \rho_{S} c_{S} / L_{n}$ is the drift frequency, $c_{s}=\sqrt{T_{e} / m_{i}}$ is the sound speed, $\rho_{s}=c_{s} / \Omega_{i}, \Omega_{i}$ is the ion gyrofrequency, and $L_{n}$ is the density scale length. Drift waves have a maximum growth rate at $k_{\perp} \rho_{s} \simeq 1$ and require finite $k_{\|}$to become unstable. ${ }^{1-3}$

The interchange instability is driven by collinear magnetic field curvature and pressure gradient. It has flute characteristics, with $k_{\|}=0$, and a global character in the perpendicular direction, growing on the largest allowed spatial scale, with growth rate $\gamma \sim c_{s} / \sqrt{R L_{p}}{ }^{3,4}$ Despite the clear differences in their character, the relative importance of these instabilities in the SOL is still a matter of debate. Experimental evidence for both mechanisms is documented in the literature and fluid models for simulation of turbulence in the SOL are run in both limits. ${ }^{5-8}$

The present paper investigates the transition from a regime dominated by drift instabilities to one dominated by pure interchange instabilities in the simple magnetized torus TORPEX. ${ }^{9}$ The TORPEX magnetic geometry consists of a toroidal magnetic field, $B_{\varphi}$, on which a vertical magnetic field, $B_{z}$, is superposed, resulting in helical field lines with both ends terminating on the torus vessel. This magnetic configuration, with open field lines characterized by long connection lengths, presents similarities with the tokamak SOL and provides a drive for both instabilities, ${ }^{10}$ allowing at the

\footnotetext{
${ }^{a)}$ Electronic mail: f.m.poli@warwick.ac.uk. Present address: Centre for Fusion, Space and Astrophysics, University of Warwick, Coventry, CV4 7AL, United Kingdom.

${ }^{b)}$ Present address: Department of Physics and Astronomy, University of California, Irvine, California 92696, USA.
}

same time more detailed diagnostics and wider parameter scans than typically possible in fusion devices.

The characterization of TORPEX instabilities in hydrogen plasmas and their development into turbulence were the subject of previous works. ${ }^{11,12}$ The case of weak vertical magnetic field was considered, in which it was shown that the observed instabilities have a drift wave character. Because of the importance of magnetic field curvature in the dispersion relation, they were referred to as drift-interchange instabilities. In the present paper we report on the transition from a regime where the drift-interchange instability has a drift character to one where it has a pure interchange character, depending on the value of the vertical magnetic field. The instabilities are analyzed and identified by studying their spectrum, their dispersion relation, and their spatial localization. Although a transition between drift and flute-type instabilities was studied in a linear device ${ }^{13}$ by varying the radius of the plasma column, and drift and interchange instabilities were observed and studied in a number of simple toroidal devices (see, for example, Refs. 10 and 14-16, and references therein), to our knowledge no evidence of the coexistence of the two instabilities in the unfavorable curvature region has been obtained so far in toroidal magnetized plasmas.

The observed transition is confirmed theoretically by the predictions of a two-fluid linear model, based on the driftreduced Braginskii equations (see, e.g., Zeiler et al. ${ }^{17}$ ). The model describes the evolution of density $n$, potential $\phi$, electron temperature $T_{e}$, and parallel current $j_{\|}$, taking into account the curvature of the magnetic field, resistivity, and finite electron mass (i.e., the drives for both the interchange and the drift instability). The model confirms that the instability has a drift character at low vertical field, and an interchange character at high vertical field.

The paper is organized as follows: After a short summary of the experimental setup and the background profiles (Sec. II), the identification of the instabilities is discussed in 
Sec. III. Following a description of the general properties of the power spectra of density and potential fluctuations for both instabilities (Sec. III A), the driving mechanism is identified by reconstructing the profile of fluctuations over the whole plasma cross section (Sec. III B). The measured dispersion relation for the interchange instability, both across and along the magnetic field, is discussed in Sec. III C, together with a comparison of the measured phase velocities with the drift velocities calculated from the background profiles. The fluid derivation of the dispersion relation is discussed in Sec. IV and the implications of these results in Sec. V.

\section{EXPERIMENTAL SETUP}

The experiments presented herein are performed in the toroidal device TORPEX (Ref. 9) (major radius $R_{0}=1 \mathrm{~m}$, minor radius $a=0.2 \mathrm{~m}$ ), in hydrogen plasmas at neutral gas pressure of $6 \times 10^{-5} \mathrm{mbar}$. The magnetic field lines are helical and result from the superposition of a dominant toroidal component, whose value is kept constant at $B_{\varphi}=76.6 \mathrm{mT}$ on axis, and a small vertical component $B_{z}$, whose magnitude is increased from 0.2 to $2 \mathrm{mT}$, in steps of $0.2 \mathrm{mT}$. This variation results in a shortening of the connection length, $L_{c}(R)=2 B_{\varphi} / B_{z} \sqrt{a^{2}-\left(R-R_{0}\right)^{2}}=2 \pi N R$, from $74 \mathrm{~m}$ to $16 \mathrm{~m}$ at $R=R_{0}$, where $N$ is the number of toroidal turns of a field line between the two points at which it intersects the vessel. Plasmas are generated and sustained by microwaves injected from the low field side, with a frequency of $2.45 \mathrm{GHz}$, in the range of the electron cyclotron frequency. Typical values for the time-averaged density, electron temperature and plasma potential are, respectively, $\bar{n} \approx 10^{16}-10^{17} \mathrm{~m}^{-3}$, $\bar{T}_{e} \approx 5 \mathrm{eV}, \bar{\phi} \approx 10-20 \mathrm{~V}$.

A four-tip Langmuir probe (LP) array, with a vertical separation of $0.3 \mathrm{~cm}$ between adjacent tips, is used to measure the radial profile of density and floating potential fluctuations at midplane, and the associated wavenumber along $z$. The same array, configured as a triple probe,${ }^{18}$ is used to measure the radial profile of temperature and electrostatic plasma fluctuations at midplane. Three arrays of eleven pairs of fixed LPs, with separation of $0.8 \mathrm{~cm}$ between pairs and $0.1 \mathrm{~cm}$ between tips within the same pair, provide measurements of density fluctuations along the azimuthal direction. The arrays, each covering an arc length of $11 \mathrm{~cm}$, are installed on the same poloidal cross section and are separated azimuthally by $\pi / 2$, to measure the dispersion relation $k_{\theta}(\omega)$ at $16 \mathrm{~cm}$ from the center of the vessel. The layout of these probes and the poloidal region covered by each array are shown in Fig. 1.

The time-averaged profiles of density, electron temperature and plasma potential, are shown in Fig. 2 for selected values of the vertical field. The plasma parameters are reconstructed in the region $\left|R-R_{0}\right| \leq 12 \mathrm{~cm}$ and $|z| \leq 13 \mathrm{~cm}$ from the $I(V)$ characteristic of an 8-tip LP array, over $0.3 \mathrm{~s}$ of stationary discharge with absorbed power of approximately $400 \mathrm{~W}$. The plasma potential profile is recovered from the temperature and the floating potential $\bar{\phi}_{f}$ as $\bar{\phi}=\bar{\phi}_{f}+\mu \bar{T}_{e} / e$, where $\mu=3.15$ is determined experimentally. ${ }^{19}$
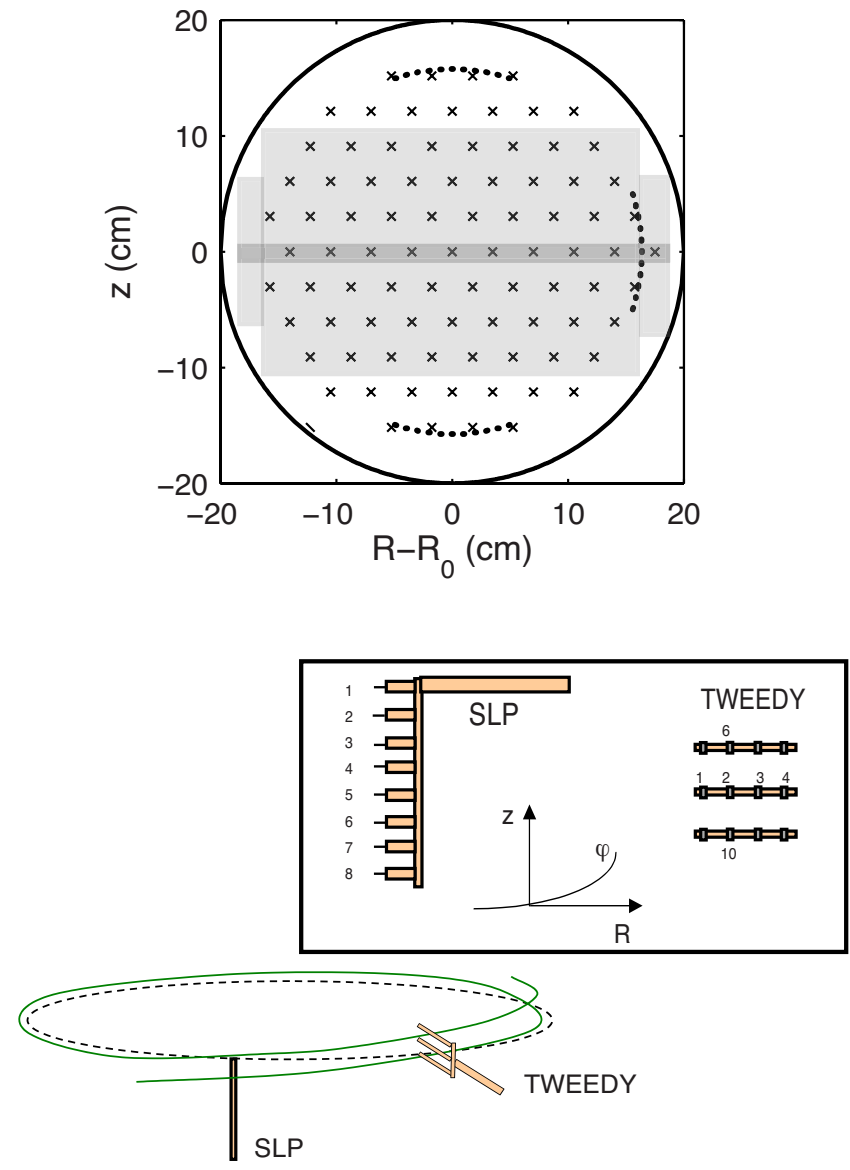

FIG. 1. (Color online) (Top) TORPEX cross section with probe positions. The shaded area indicates the region covered by the 8-tip probe array for the measurement of plasma profiles. Crosses represent the position of an 86-tip array for the measurement of density and floating potential fluctuations. The three arrays of 11 pairs of probes are indicated as dots. The dark shaded area indicates the region of measurement of the 4-tip probe array used for the measurement of the dispersion relation at midplane. (Bottom) Configuration used for the measurement of the parallel wavenumber. The dotted curve represents the equatorial plane, while the continuous line sketches a magnetic field line intersecting the two arrays of probes.

The density profile is centered with respect to the vessel cross section and has good symmetry along $R$ and $z$. Temperature and plasma potential profiles are more elongated in the $z$ direction and approach a slablike configuration with increasing vertical field magnitude. The maximum density stays approximately constant, with relative variations below $20 \%$ across the full range of the vertical field values; the relative increase in temperature and plasma potential is twice as large.

Figure 3 shows the radial gradient scale lengths for density and temperature, defined as $L_{n}=\bar{n} / \nabla_{R} \bar{n}$ and $L_{T}=\bar{T}_{e} / \nabla_{R} \bar{T}_{e}$, and of the background electric field as a function of the vertical magnetic field. The plotted values correspond to measurements taken on the low field side, at the radial positions of the maximum gradients of $\bar{n}, \bar{T}_{e}$, and $\bar{\phi}$, which coincide at $6 \mathrm{~cm}$ within the experimental uncertainties. The density gradient scale length stays approximately constant up to $B_{z}=1.2 \mathrm{mT}$ and is comparable to the temperature gradient scale length for all values of the vertical field. 


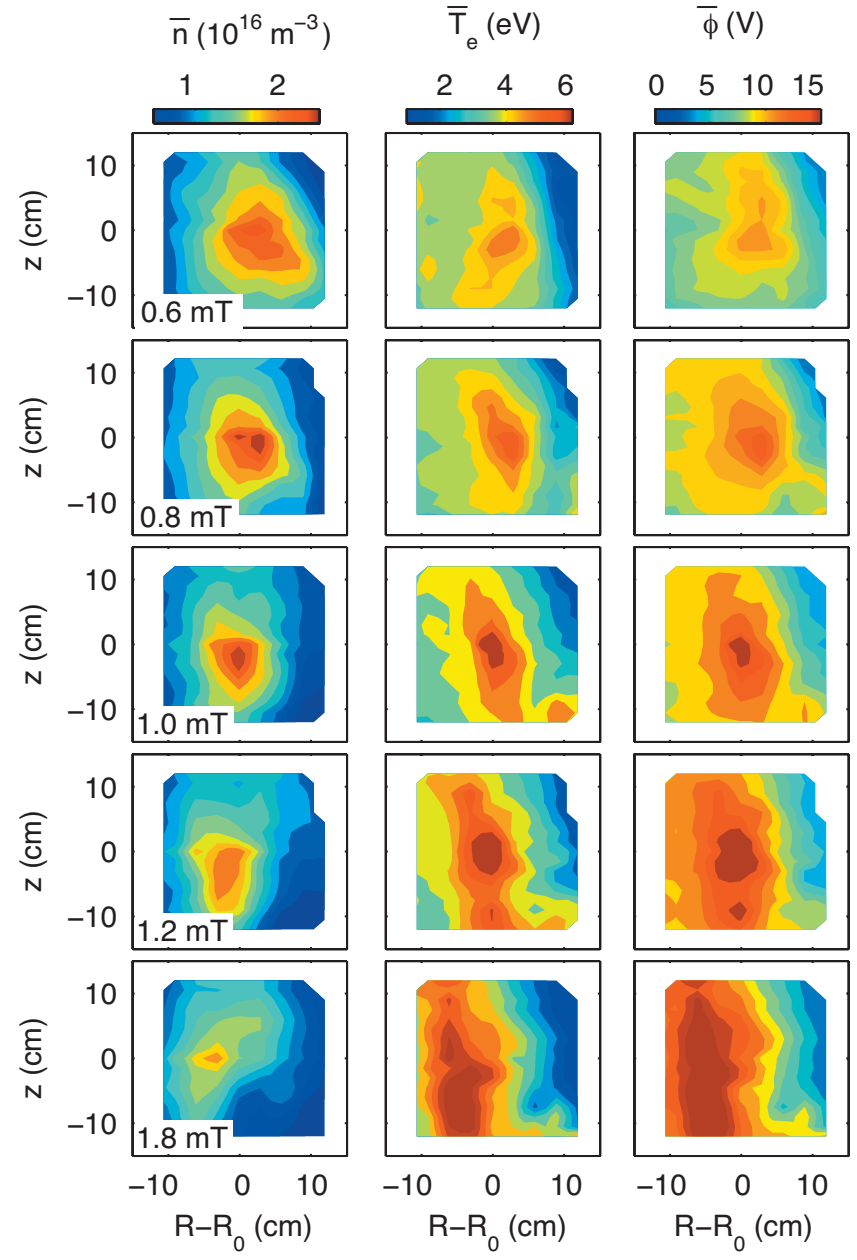

FIG. 2. (Color) Time averaged profiles of density, electron temperature, and plasma potential, for different values of the vertical field, increasing from top to bottom.

Comparable values of $L_{n}$ and absence of a clear trend with $B_{z}$ are also observed on the plasma high field side.

We note that, in agreement with Fig. 3(a), Ref. 8 predicts, for the turbulent regime typical of these experiments, that the density and temperature scale lengths remain constant when the magnetic field is varied. Although the maximum value of the electrostatic plasma potential monotonically increases with $B_{z}$, the electric field varies between 60 and $150 \mathrm{~V} / \mathrm{m}$, without a clear trend.

\section{IDENTIFICATION OF THE INSTABILITIES}

A complete characterization of the nature of instabilities is performed on TORPEX by comparing the measurements of density and potential fluctuations taken at different locations over the whole plasma cross section. From the power spectrum of $\tilde{n}$ over the whole plasma cross section, we reconstruct the profile of the amplitude of fluctuations associated with coherent spectral components (see, for example, Refs. 11 and 20 for a detailed description of the method). Assuming that the profile of the mode amplitude is related to that of the corresponding instability drive, this method allows us to identify the driving mechanism and to separate distinct driving mechanisms associated with individual spec-

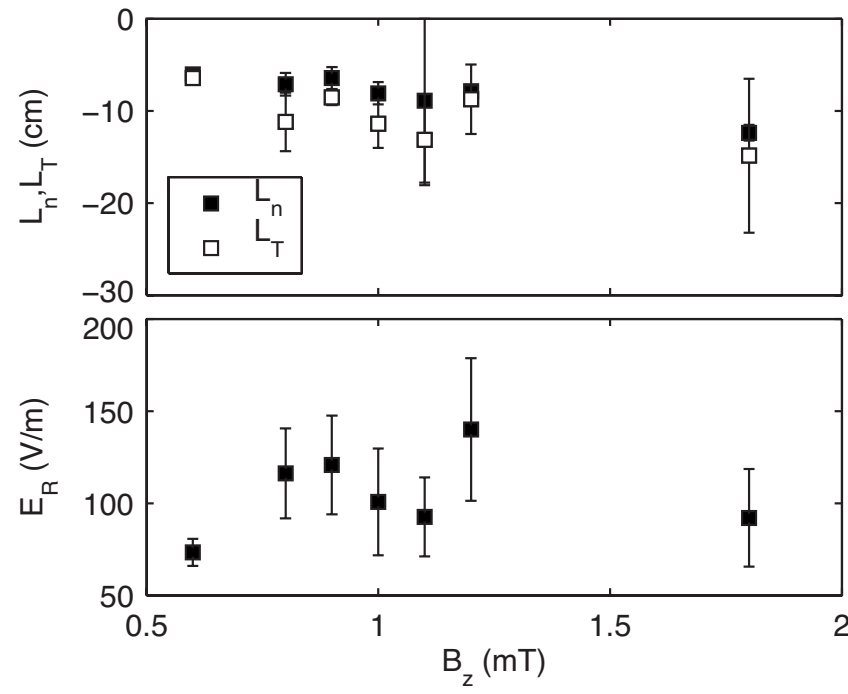

FIG. 3. Top: Variation of the density gradient scale length $L_{n}$ (black squares) and of the electron temperature gradient scale length $L_{T}$ (open squares) with the amplitude of the vertical field, measured on the low field side, at the position of maximum pressure gradient. Negative values of $L_{n}$ and $L_{T}$ indicate that the associated gradients decrease along positive $R$. Bottom: Variation of the radial electric field with $B_{z}$, measured at the same location.

tral components. The second step is the measurement of the dispersion relation both across and along the magnetic field. In the latter case, care is taken to keep probes aligned along the magnetic field lines. ${ }^{11,20}$

We do not rely on the measurement of the phase shift between $\tilde{n}$ and $\tilde{\phi}$ for a definite identification of the nature of the instabilities, nor on the comparison between the normalized density and potential fluctuations. The value of the phase shift between $\tilde{n}$ and $\tilde{\phi}$ is commonly used in plasma experiments to discriminate between drift instabilities (phase shift between 0 and $\pi / 4$ ) and flute instabilities (phase shift between $\pi / 2$ and $\pi){ }^{21}$ On TORPEX the phase shift between density and potential fluctuations does not seem to depend unequivocally on the nature of the instability, as will be discussed in Sec. III A, and it cannot be used as a discriminating quantity.

\section{A. Power spectrum of fluctuations}

The radial profile of density and potential fluctuations measured at midplane, as well as the associated power spectra, are shown in Fig. 4 for $B_{z} \in[0.6,1.8] \mathrm{mT}$. The spectra refer to measurements taken on the high field side, at $R$ $-R_{0}=-9 \mathrm{~cm}$, and on the low field side, at the position of maximum level of density fluctuations, approximately equal to $6 \mathrm{~cm}$ for most values of the vertical magnetic field. Density fluctuations peak on the low field side with respect to the maximum of $\bar{n}$, at the position of maximum pressure gradient, a feature common to a wide variety of TORPEX experimental scenarios. ${ }^{11,9}$ At this location $\tilde{n} / \bar{n} \approx 90 \%$ for most values of $B_{z}$. Plasma potential fluctuations are estimated as $\widetilde{\phi}=\widetilde{\phi}_{f}+\mu \widetilde{T}_{e} / e$, where $\widetilde{\phi}_{f}$ and $\widetilde{\phi}$ denote, respectively, the fluctuating part of the floating and of the plasma potential. The normalized values of density and potential fluctuations, integrated over the whole range of frequencies, are comparable 

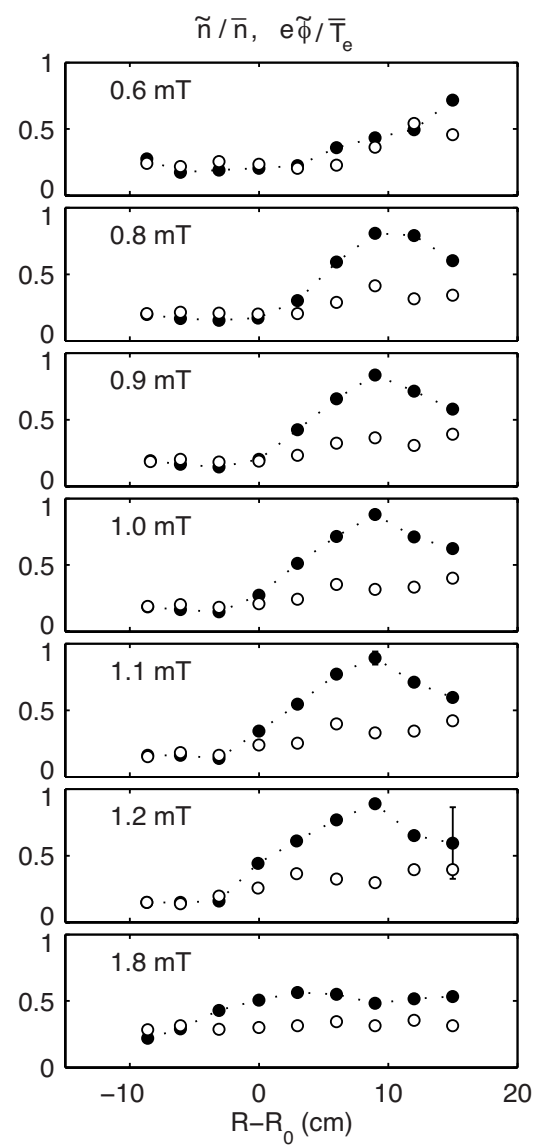

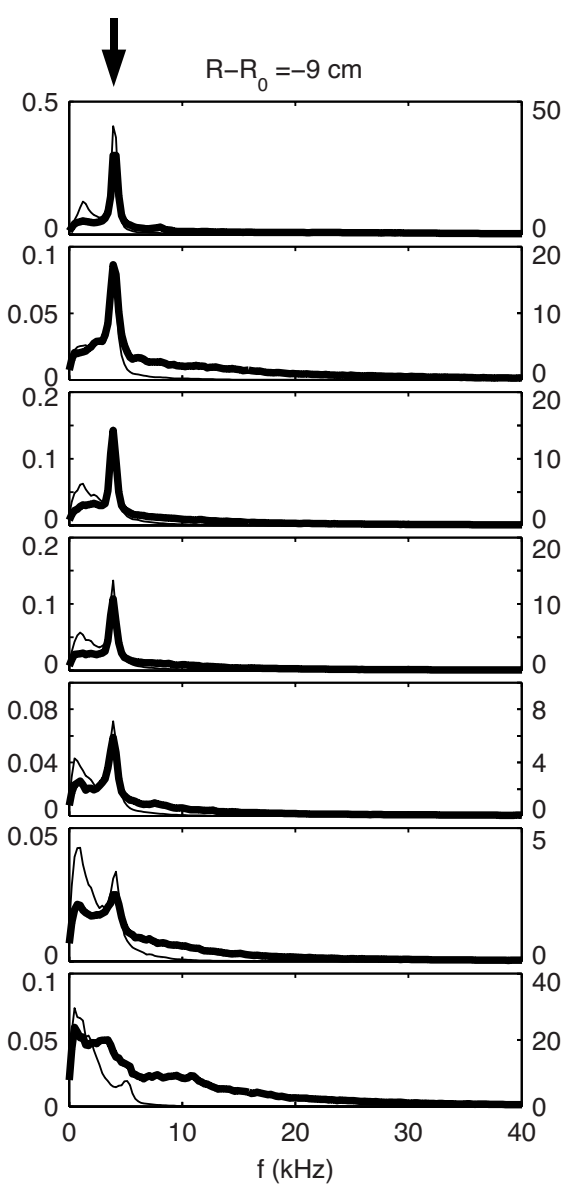

FIG. 4. Left: Radial profile of $\tilde{n} / \bar{n}$ (black circles) and $\widetilde{\phi} / \bar{T}_{e}$ (open circles), for different values of $B_{z}$, increasing from top to bottom, as indicated in the legend. Center: power spectrum (a.u.) of $\tilde{n}$ (thick line, left axis) and $\widetilde{\phi}$ (thin line, right axis) measured on the high field side. Right: as the center column, but for measurements on the low field side. The drift-interchange mode is indicated by the black arrows on the top of the picture, while the interchange mode is indicated by the shaded stripe in the right column.

in amplitude on the high field side for all values of $B_{z}$, while $\tilde{n} / \bar{n} \approx 2 e \widetilde{\phi} / \bar{T}_{e}$ on the low field side for $B_{z}>0.6 \mathrm{mT}$.

Two low frequency modes with different properties are detected in the power spectra of $\tilde{n}$. The first one, with frequency $\approx 4 \mathrm{kHz}$, is measured both on the high and on the low field side, and its frequency and amplitude are barely affected by the value of $B_{z}$. The second one is measured only on the low field side, in a layer of radial extension of 5-8 cm, and its spectral features are strongly affected by the magnitude of the vertical field. The peak associated with this second mode is broad in frequency at the lowest $B_{z}$, and becomes narrower with increasing vertical field magnitude. Its frequency and its spectral width decrease, respectively, from 15 to $5 \mathrm{kHz}$ and from 8 to $0.8 \mathrm{kHz}$ when $B_{z}$ is raised from 0.8 to $1.8 \mathrm{mT}$. At the same time the amplitude increases and this mode dominates the power spectrum of fluctuations for $B_{z}>1.0 \mathrm{mT}$. These differences in the spectral features suggest that the two modes are associated with instabilities of a different nature. The dispersion properties of the instability at $4 \mathrm{kHz}$ have been analyzed in detail for $B_{z}=0.6 \mathrm{mT}$ and the instability was identified as a drift-interchange. ${ }^{11} \mathrm{On}$ the HFS this mode is narrow in frequency and wavenumber for $R-R_{0}<-10 \mathrm{~cm}$, close to the location of the EC resonance layer at $-12.5 \mathrm{~cm}$. On the left of the $\mathrm{EC}$ resonance layer the density gradient is even steeper than that measured on the LFS and can destabilize drift waves. ${ }^{19,11}$ The density gradient at the EC resonance layer was shown to be independent of external parameters, such as the neutral gas pressure and the vertical magnetic field. ${ }^{19}$ This, in addition to the fact that the $\mathbf{E} \times \mathbf{B}$ velocity on the HFS is negligible with respect to the diamagnetic drift velocity, justifies why the measured wavenumbers and frequencies hardly change with the vertical field. Since the vertical and radial wavelengths associated with the mode are comparable to or larger than the radial extension of the EC resonance layer, we cannot exclude that the small region of unfavorable curvature present on the right of the EC resonance layer may further contribute to destabilizing the mode. The density fluctuations associated with the instability have their maximum amplitude in a limited region at the bottom of the plasma cross section, on the low field side, as also shown in Fig. 6. This suggests that the driftinterchange instability is convected to this location from the HFS. Following the convection motion on the LFS, the amplitude of the mode decreases, mostly because of nonlinear interactions that transfer energy to other spectral components. ${ }^{12}$ The wave dispersion barely changes with increasing vertical field, with a phase velocity in the range of $600-1000 \mathrm{~m} / \mathrm{s}$, and parallel and perpendicular wavenum- 


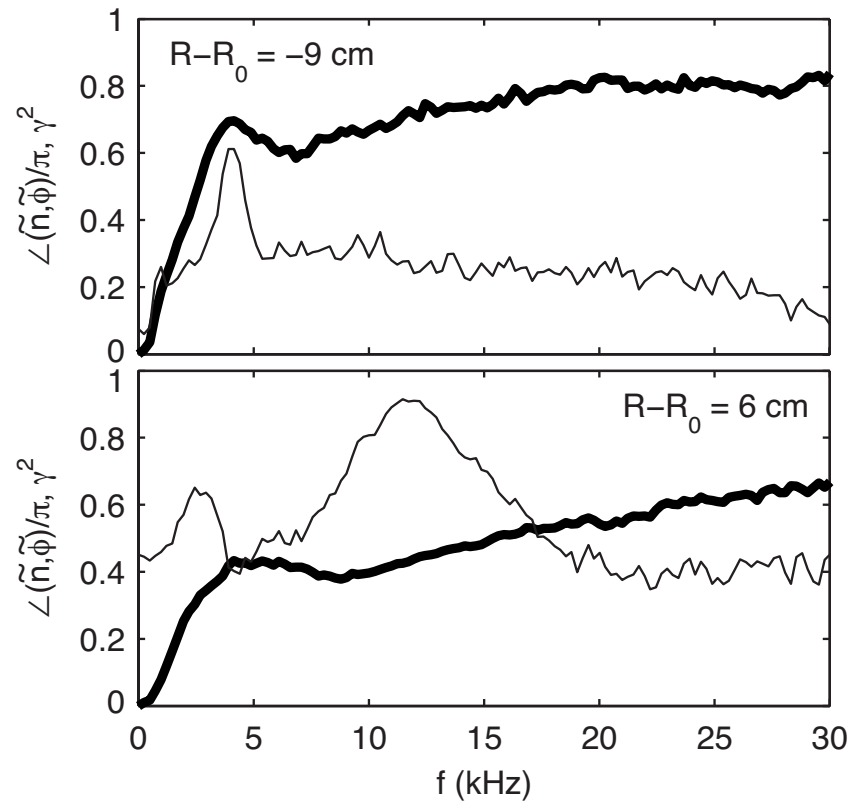

FIG. 5. Phase shift between density and plasma potential fluctuations (thick line), measured on the high (top) and on the low field side (bottom), for $B_{z}=1.2 \mathrm{mT}$. The coherence spectrum (thin line) is shown for comparison.

bers, respectively, equal to $k_{\|}=0.3-0.4 \mathrm{~m}^{-1}$ and $k_{z}=15$ $-30 \mathrm{~m}^{-1}$. The drift character of the instability is confirmed for all the considered values of the vertical field, though details of the measurements are not discussed in this paper.

We focus instead on the identification of the second instability and on the transition between the two regimes. It is shown below that the mode detected on the low field side is an interchange instability, driven by the curvature of the magnetic field in the region where the magnetic field gradient and the pressure gradient are collinear. The interchange instability was found to have an important role in the generation of blobs on TORPEX, which detach from the main plasma and propagate radially outward..$^{22,23}$ The blob scenario was characterized for values of the vertical magnetic field larger than those analyzed in this paper and for a slightly lower value of the neutral gas pressure. Nevertheless, the measured frequency, $4 \mathrm{kHz}$, was lower than the value we measure at $1.8 \mathrm{mT}$ and consistent with the trend observed with $B_{z}$. The interchange nature of the instability was also confirmed by a comparison with a two-fluid interchange model.

Figure 4 suggests that interchange and drift-interchange instabilities can coexist on the low field side of the maximum of $\bar{n}$ for some values of the vertical field. The amplitude of the interchange mode is dominant over that of the driftinterchange mode for $B_{z}>1.0 \mathrm{mT}$, where the latter can no longer be detected at midplane. As noted above, the normalized density fluctuations are approximately twice as large as the normalized potential fluctuations on the low field side. These differences are mostly due to the interchange instability detected on the low field side. The normalized density and potential fluctuations are in fact comparable for $B_{z}<0.8 \mathrm{mT}$, where the interchange instability has very low amplitude, but differ in value for $B_{z} \geq 0.8 \mathrm{mT}$, where the interchange mode dominates the power spectrum of $\tilde{n}$.
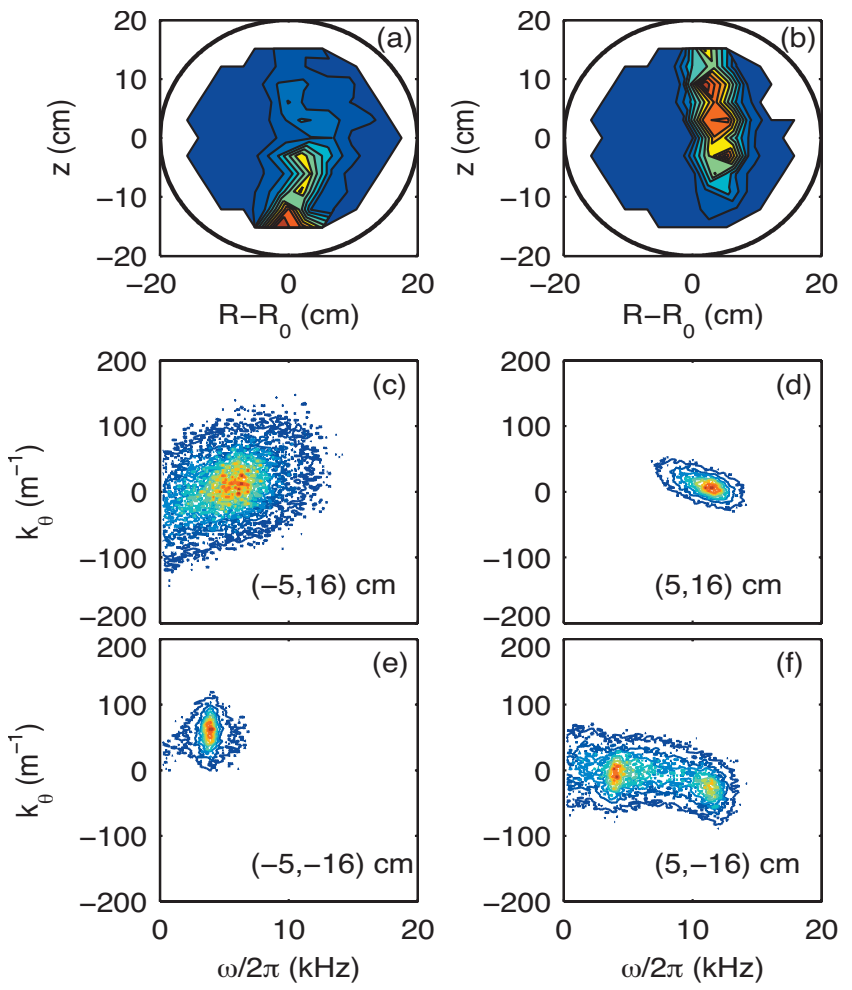

FIG. 6. (Color) Top: Profile of the rms value of density fluctuations associated with the drift-interchange instability (a) and with the interchange instability (b), for $B_{z}=1.2 \mathrm{mT}$. Middle: $P_{n}\left(k_{\theta}, \omega\right)$, measured at the upper part of the poloidal cross section, on the high (c) and on the low (d) field side. Bottom: $P_{n}\left(k_{\theta}, \omega\right)$, at the lower part of the poloidal cross section, on the high (e) and on the low (f) field side.

The phase shift between density and potential fluctuations is shown in Fig. 5 for the case of $B_{z}=1.2 \mathrm{mT}$. For both instabilities, the measured phase shift is close to $\pi / 2$, thus preventing a clear identification of the nature of the instabilities on the basis of this quantity. Also for the other vertical field values the phase shift varies between $+\pi$ and $-\pi$, with no clear separation between the two instabilities. Note that the value of the phase shift between $\widetilde{n}$ and $\widetilde{\phi}$ has direct implications on the value of the fluctuation-induced particle flux. If it is independent of the nature of the instabilities, one cannot define "typical" particle fluxes associated with instabilities of a different nature. Experiments on TORPEX have demonstrated in fact that interchange and drift instabilities give comparable values of the fluctuation induced particle flux related to fluctuations in the range of vertical magnetic field values between 0.9 and $1.2 \mathrm{mT}^{24}$

\section{B. Identification of the driving mechanism}

The drift-interchange and the interchange instability are separated in frequency and appear at two separate locations, as it is evident from the measurement of $P\left(k_{\theta}, \omega\right)$, shown in Fig. 6 for the case of $B_{z}=1.2 \mathrm{mT}$. A comparison of the spectra, measured at the upper (Figs. 6(c) and 6(d)) and at the lower (Figs. 6(e) and 6(f)) of the poloidal cross section, confirms that the interchange mode is detected only in the unfavorable curvature region, while the drift-interchange mode is detected on both sides. Figures 6(a) and 6(b) show the 2D 

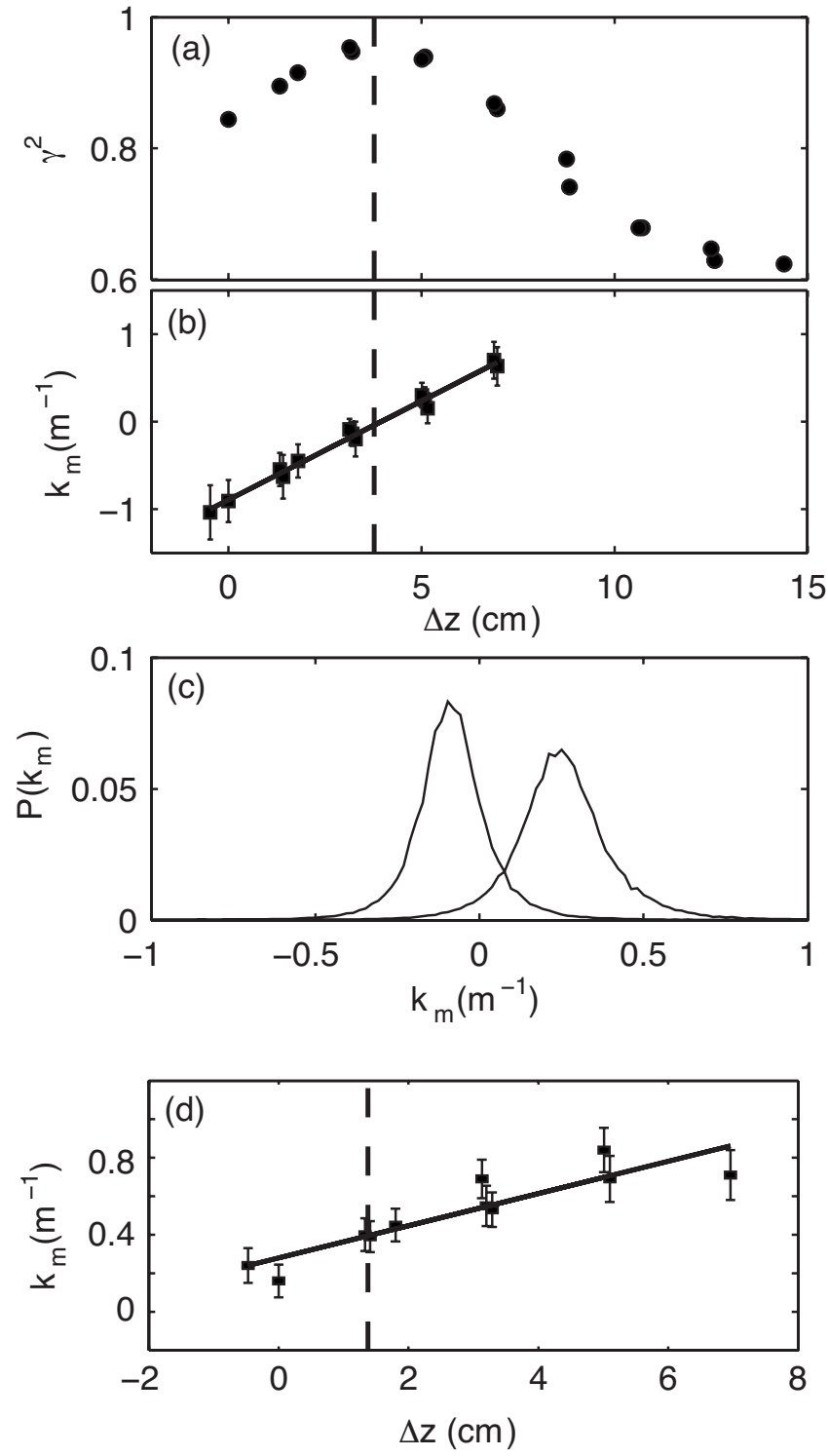

FIG. 7. (a-c) Wavenumber measured at $R-R_{0}=5 \mathrm{~cm}$, for $B_{z}=1.6 \mathrm{mT}$. (a) Amplitude of the coherence spectrum, calculated at the frequency of the mode as a function of the vertical separation between tips, calculated with respect to the tip in the downstream array. The dashed line indicates the vertical coordinate $z_{0}$, where the magnetic field line that passes through the reference tip at the location of the downstream probe intersects the upstream array. (b) Measured wavenumber as a function of the vertical separation between tips. The plotted value of $k_{m}$ is the center of the Gaussian curve that fits the wavenumber spectrum $P\left(k_{m}\right)$, the error bar is its spectral width. (c) Wavenumber spectrum measured around $z_{0}$, at $\Delta z \simeq 3 \mathrm{~cm}\left(k_{m}=-0.1 \mathrm{~m}^{-1}\right)$, and $\Delta z=5 \mathrm{~cm}\left(k_{m}=0.22 \mathrm{~m}^{-1}\right)$. (d) Wavenumber measured at $R-R_{0}=9 \mathrm{~cm}$, for $B_{z}=0.6 \mathrm{mT}$.

profile of the rms value of density fluctuations associated with the two instabilities for $B_{z}=1.2 \mathrm{mT}$. The rms fluctuations are reconstructed as $\left[P\left(\omega_{0}\right) \Delta \omega_{0}\right]^{1 / 2}$, where $P\left(\omega_{0}\right)$ is the power spectral density of $\tilde{n}$ at frequency $\omega_{0}$ and $\Delta \omega_{0}$ is the mode spectral width. Both instabilities have their maximum amplitude on the low field side, with the drift-interchange instability being maximum at the lower part of the plasma cross section, ${ }^{11,12}$ and the interchange instability being maximum in a region extended along the $z$ direction.

The spectral measurements, taken over the whole poloidal cross section, confirm that the interchange mode is gen-
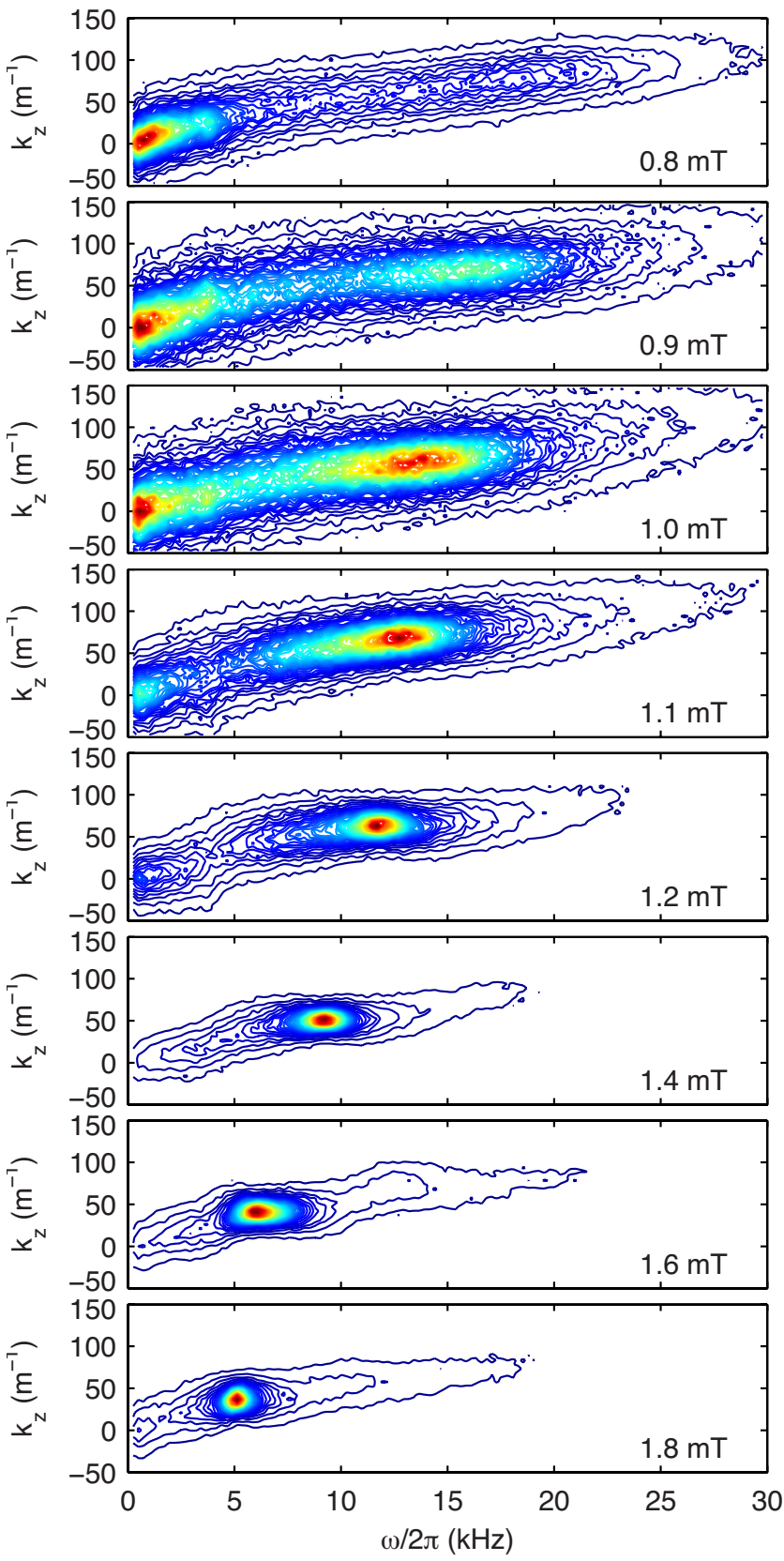

FIG. 8. (Color online) Wavenumber and frequency spectrum of $\widetilde{n}$, measured at midplane from two LPs with vertical separation of $0.6 \mathrm{~cm}$, as a function of the vertical field, increasing from top to bottom. Measurements have been taken for each value of $B_{z}$ at the radial position of the maximum level of fluctuations. The value of $P_{n}\left(k_{z}, \omega\right)$ is normalized to the total power, $\Sigma_{k_{z}, \omega} P_{n}\left(k_{z}, \omega\right)$.

erated and survives only on the low field side, where the curvature of the magnetic field is destabilizing. The driftinterchange instability is instead convected to the unfavorable curvature region where the curvature acts as an additional destabilizing factor.

\section{Measurement of the dispersion relation}

The dispersion relation across and along the magnetic field is extracted from the wavenumber-resolved power spectrum associated with density fluctuations, $P_{n}(k, \omega)$. The latter is reconstructed by applying a two-point correlation 


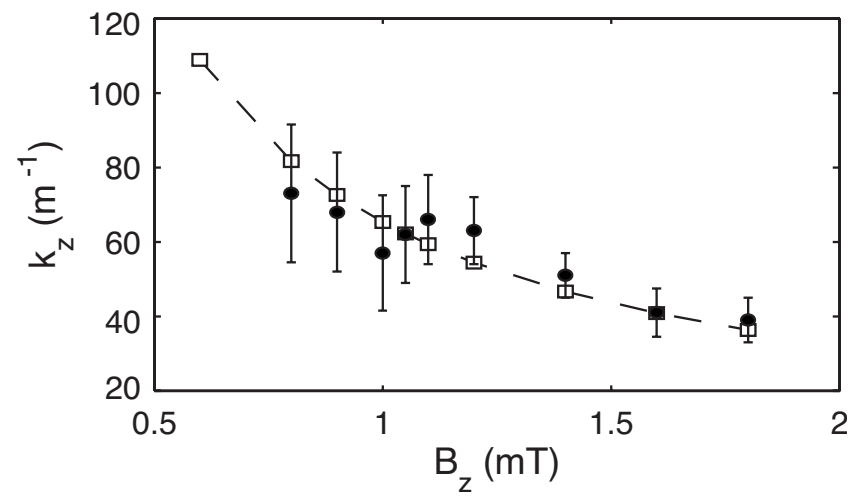

FIG. 9. Variation of $k_{z}$ (black circles) of the interchange mode with the $B_{z}$. The vertical bar represent the spectral width in the wavenumber space. The open circles represent the wavenumber calculated as $2 \pi / \Delta$, where $\Delta$ is the vertical displacement of a field line after a complete toroidal turn.

technique $^{25}$ to the time series of $\tilde{n}$ measured with two Langmuir probes, with mutual separation typically much smaller than the related correlation length.

The parallel wavenumber has been measured from the phase shift between two probes with toroidal separation of $\pi / 2$, positioned at the same radial location. Because of the effect of the projection of the perpendicular wavenumber $k_{\perp}$ along the direction of measurement, when probe tips are not exactly aligned along the same field line, the measured wavenumber is a combination of $k_{\perp}$ and $k_{\|}$. We have used two arrays of probes and we have measured the phase shift for all combinations of tips belonging to different arrays. ${ }^{20}$ Figure 7 shows the results of the measurements for $B_{z}=1.6 \mathrm{mT}$. The value of the coherence spectrum measured at the frequency of the mode, $\omega / 2 \pi \simeq 9 \mathrm{kHz}$, is maximum at a coordinate $z$ close to the calculated position of the same magnetic field line, $z_{0}$, indicated in the figure with a dashed line. The measured wavenumber $k_{m}$ changes its sign across $z_{0}$, suggesting that $k_{\|}$is close to zero; only an upper estimate of $k_{\|}$can be given on the basis of the measured phase shift. The wavenumber measured from the spectrum $P\left(k_{m}\right)$ at the coordinate where the coherence is maximum is $0.12 \mathrm{~m}^{-1}$, with a width of $0.14 \mathrm{~m}^{-1}$, Fig. 7(c). An interpolation of the measured $k_{m}$ at the coordinate $z_{0}$, using the parameters of a linear fit, ${ }^{11}$ gives $k_{\|} \simeq 0.06 \mathrm{~m}^{-1}$. The same sign inversion in $k_{m}$ is observed for all values of $B_{z}$, indicating that the instability detected on the low field side has always an interchange character. We note that this sign inversion is not observed in the case of the drift-interchange instability, as shown in Fig. 7(d).

Figure 8 shows $P_{n}\left(k_{z}, \omega\right)$, measured at midplane with two Langmuir probes, located at the same radial and toroidal positions, and separated by $0.6 \mathrm{~cm}$ along $z$. The spectra shown in the figure refer to measurements taken on the low field side and, for each value of $B_{z}$, at the position of the maximum level of density fluctuations. Although for low values of $B_{z}$ the frequency spectrum is broader, a wavenumber $k_{z}$ can still be clearly identified. For increasing vertical field amplitude the spectrum becomes narrower both in the $\omega$ - and in the $k_{z}$-space and peaks at lower values of frequency and wavenumber. For all values of $B_{z}$ we measure $\sigma_{k_{z}}<k_{z}$, indi-
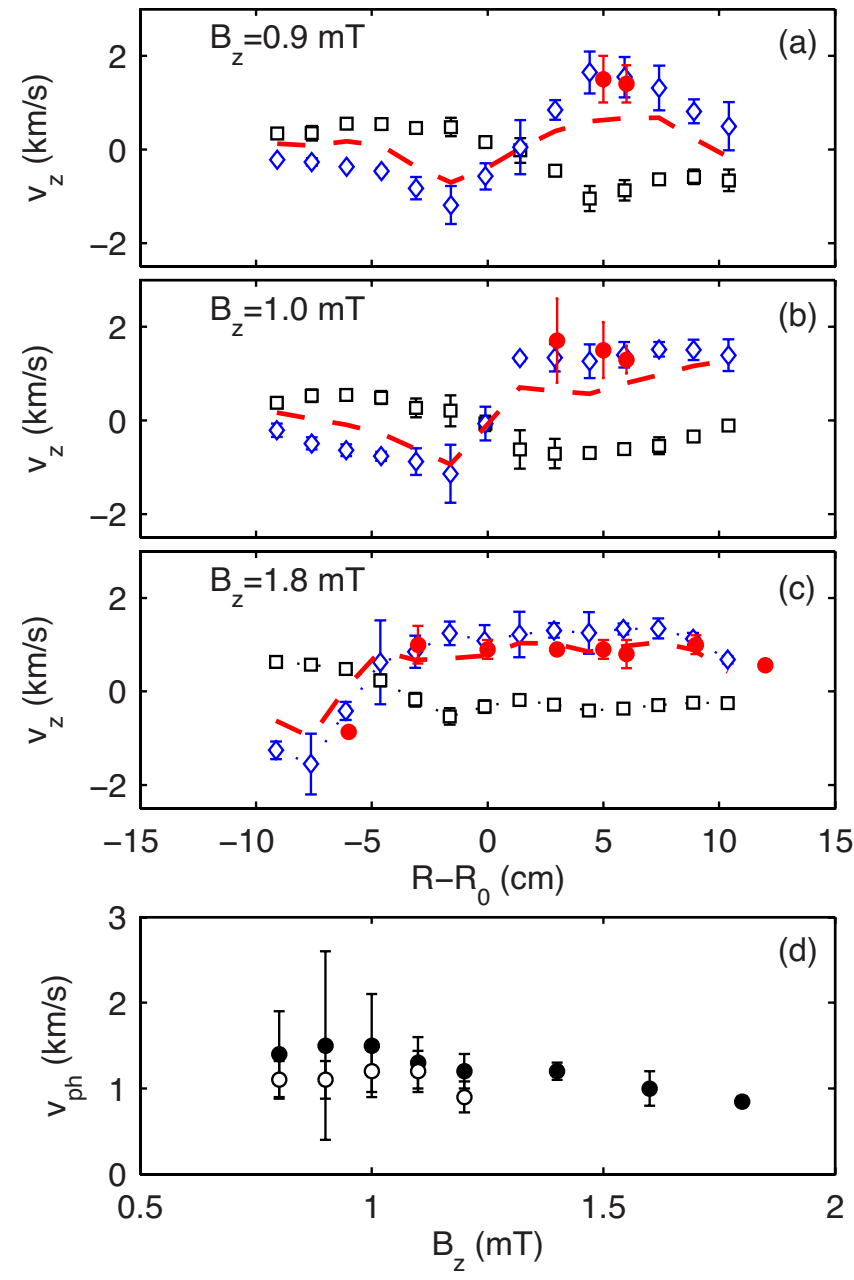

FIG. 10. (Color online) (a-c) Radial profile of the phase velocity (red circles), $\omega / k_{z}$, measured at midplane, for different values of the vertical field. Squares indicate the vertical component of the electron diamagnetic drift velocity, $\mathbf{v}_{* e} \cdot \hat{\mathbf{z}}$, while diamonds indicate the $\mathbf{E} \times \mathbf{B}$ drift velocity, $\mathbf{v}_{\mathrm{E}} \cdot \hat{\mathbf{z}}$. The component along $\hat{z}$ of the difference $\left|\mathbf{v}_{E}\right|-\left|\mathbf{v}_{* e}\right|$ is indicated with a dashed red line. (d) Measured phase velocity of the drift-interchange mode (open circles) and of the interchange mode (black circles) as a function of the vertical field magnitude.

cating that turbulence is weakly developed even for the lowest vertical field values. The correlation length, estimated as the inverse of the spectral width in $k_{z}$, increases from 17 to $48 \mathrm{~cm}$ when $B_{z}$ is raised from 0.8 to $1.8 \mathrm{mT}$. The variation of $k_{z}$ with the vertical field magnitude is shown in Fig. 9. The measured wavenumbers are in good agreement with the inverse wavelength corresponding to a complete toroidal turn, $\Delta=2 \pi R_{1} B_{\mathrm{z}} / B_{\varphi}$, where $R_{1}=R_{0}+6 \mathrm{~cm}$ is the radial position where $k_{z}$ is measured. This is the wavelength expected for an interchange instability, as detailed in Sec. IV. Larger values of $B_{z}$ correspond to shorter connection lengths, and are thus associated with longer perpendicular wavelengths, i.e., smaller values of $k_{z}$.

Figures 10(a)-10(c) show the radial profile of $\omega / k_{z}$, measured at midplane, for $B_{z}=0.9,1.0$, and $1.8 \mathrm{mT}$. For each $B_{z}$ value, the measured phase velocity is compared with the radial profile of the electron diamagnetic and of the $\mathbf{E} \times \mathbf{B}$ drift velocity, resp. $\mathbf{v}_{* e}$ and $\mathbf{v}_{E}$. As the background density gradient and the electric field are at midplane along $R$, the 
component along $z$ of $\mathbf{v}_{* e}$ and $\mathbf{v}_{E}$ is represented in the picture. As $\mathbf{v}_{E, z}$ and $\mathbf{v}_{* e, z}$ have opposite directions, the resulting velocity at midplane is the difference between their absolute values. Over most of the radial profile $\left|\mathbf{v}_{* e}\right|<\left|\mathbf{v}_{E}\right|$ for all values of $B_{z}$, and the difference between the two is close to $\left|\mathbf{v}_{E}\right|$. As the interchange instability is measured only on the low field side, the comparison between the phase velocity and the drift velocities is possible only in a limited region of the equatorial plane. The wave frequency is measured in the laboratory frame, thus is Doppler shifted by the $\mathbf{E} \times \mathbf{B}$ frequency, $k_{z} v_{E}$. For the typical TORPEX background parameters, the phase velocity of a pure interchange instability is below $100 \mathrm{~m} / \mathrm{s}$, thus approximately 20 times lower than the $\mathbf{E} \times \mathbf{B}$ velocity. The phase velocity measured in the laboratory frame is in this case essentially the $\mathbf{E} \times \mathbf{B}$ velocity. The measured phase velocity is in good agreement with $\mathbf{v}_{E}$ for $B_{z}=0.9 \mathrm{mT}$ and $B_{z}=1.0 \mathrm{mT}$. For $B_{z}=1.8 \mathrm{mT},\left|\mathbf{v}_{* e}\right| \ll\left|\mathbf{v}_{E}\right|$, and it is difficult to conclude whether the measured phase velocity corresponds to $\left|\mathbf{v}_{E}\right|$ or to $\left|\mathbf{v}_{E}\right|-\left|\mathbf{v}_{* e}\right|$.

For the values of $B_{z}$ for which both instabilities are observed on the low field side, the phase velocity of the driftinterchange mode is, on average, $300 \mathrm{~m} / \mathrm{s}$ lower than that of the interchange mode, as shown in Fig. 10(d). This difference is comparable in amplitude to $\left|\mathbf{v}_{* e}\right|$, as calculated from the background profiles, although the large error bars in the measured electric field prevent us from a more detailed comparison between the phase velocities of the two instabilities and the drift velocities.

Once the value of the wavenumber is fixed from the geometry, as discussed above, the measured frequency is the $\mathbf{E} \times \mathbf{B}$ frequency, $\omega_{E}=k_{z} v_{E}$, in agreement with the observed trend of frequency and wavenumber with the vertical field, Fig. 8.

\section{COMPARISON WITH THEORY}

We consider the linearized drift-reduced Braginskii equations (see, e.g., Ref. 17) in the limit $\beta=2 \mu_{0} \bar{n} \bar{T}_{e} / B^{2} \ll 1$, $T_{i} \ll T_{e}, \quad v_{\|, i} \ll v_{\|, e}$. The equations are written in a fieldaligned coordinate system, where $R$ is the radial direction and $y$ is the direction perpendicular to both $R$ and the parallel direction. It should be noted that $y$ coincides with the cylindrical $z$ coordinate only for a purely toroidal magnetic field.

By neglecting stationary flows (i.e., $\bar{v}_{\|, e}=0, \bar{\phi}=0$ ), the linearized equations for the perturbed quantities $\widetilde{n}, \widetilde{\phi}, \widetilde{T_{e}}$, and $\tilde{j}_{\|}$are

$$
\frac{\partial \widetilde{n}}{\partial t}=-\frac{1}{B} \frac{\partial \widetilde{\phi}}{\partial y} \frac{\partial \bar{n}}{\partial R}+\frac{2}{e R_{1} B}\left(\bar{n} \frac{\partial \widetilde{T}_{e}}{\partial y}+\bar{T}_{e} \frac{\partial \widetilde{n}}{\partial y}-e \bar{n} \frac{\partial \widetilde{\phi}}{\partial y}\right)+\frac{\nabla_{\|} \tilde{j}_{\|}}{e},
$$

$$
\frac{\partial \nabla^{2} \widetilde{\phi}}{\partial t}=\frac{2 B}{m_{i} R_{1}}\left(\frac{\bar{T}_{e}}{\bar{n}} \frac{\partial \widetilde{n}}{\partial y}+\frac{\partial \widetilde{T}_{e}}{\partial y}\right)+\frac{B \omega_{c i}}{e n} \nabla_{\| \tilde{j}_{\|}},
$$

$$
\begin{aligned}
\frac{\partial \widetilde{T}_{e}}{\partial t}= & -\frac{1}{B} \frac{\partial \widetilde{\phi}}{\partial y} \frac{\partial \bar{T}_{e}}{\partial R}+\frac{4}{3 e R_{1} B}\left(\frac{7}{2} \bar{T}_{e} \frac{\partial \widetilde{T}_{e}}{\partial y}+\frac{\bar{T}_{e}^{2}}{\bar{n}} \frac{\partial \widetilde{n}}{\partial y}-e \bar{T}_{e} \frac{\partial \widetilde{\phi}}{\partial y}\right) \\
& +\frac{2}{3} \frac{\bar{T}_{e}}{\bar{n} e} 1.71 \nabla_{\|} \tilde{j}_{\|} \\
\frac{m_{e}}{e^{2} \bar{n}} \frac{\partial \tilde{j}_{\|}}{\partial t} & =-\frac{\tilde{j}_{\|}}{\sigma_{\|}}-\nabla_{\|} \widetilde{\phi}+\frac{\bar{T}_{e}}{e \bar{n}} \nabla_{\|} \tilde{n}+\frac{1.71}{e} \nabla_{\|} T_{e} .
\end{aligned}
$$

It is defined ${ }^{26}$ as $\sigma_{\|}=1.96 n e^{2} \tau_{e} /\left(4 \pi \epsilon_{0} m_{e}\right)$ and $\tau_{e}$ $=6 \sqrt{2 m_{e}}\left(\pi k_{B} T_{e}\right)^{3 / 2} \epsilon_{0}^{2} /\left(n \Lambda e^{4}\right)$, where $\Lambda$ is the Coulomb logarithm. Assuming that the perturbations do not depend on $R$ and are expressed in the form $\tilde{f}=\tilde{f} \exp \left(\gamma t+i k_{y} y+i k_{\|} x_{\|}\right)$, the dispersion relation associated with Eqs. (1)-(4) can be written as

$$
a_{0}+a_{1} \gamma+a_{2} \gamma^{2}+a_{3} \gamma^{3}+a_{4} \gamma^{4}=0
$$

with

$$
\begin{aligned}
a_{0}= & \frac{2}{3}\left[10 i \omega_{d} \nu-6.01 \omega_{\|}^{2}\right] \omega_{d}\left(2 \omega_{d}+\omega_{*}\right) \\
a_{1}= & \frac{1}{3} i\left(40 \frac{m_{e}}{m_{i}} \omega_{d}^{3}-20\left[i\left(k_{y}^{2}-1\right) \nu-\frac{m_{e}}{m_{i}} \omega_{*}\right] \omega_{d}^{2}\right. \\
& +2\left\{\left[5.85 k_{y}^{2}+7 k_{y}^{2}-6.84\left(k_{y}^{2}+1\right)+4\right] \omega_{\|}^{2}\right. \\
& \left.\left.+3 i(\eta+1) \nu \omega_{*}\right\} \omega_{d}-3(1.71 \eta+1) \omega_{\|}^{2} \omega_{*}\right), \\
a_{2}= & \frac{1}{3}\left\{20\left(k_{y}^{2}-1\right) \frac{m_{e}}{m_{i}} \omega_{d}^{2}+\left[20 i \nu k_{y}^{2}-6 \frac{m_{e}}{m_{i}}(\eta+1) \omega_{*}\right] \omega_{d}\right. \\
& \left.-\left[8.85 k_{y}^{2}+3\right] \omega_{\|}^{2}\right\}, \\
a_{3}= & \frac{1}{3} k_{y}^{2}\left(20 i \omega_{d} \frac{m_{e}}{m_{i}}-3 \nu\right) \\
a_{4}= & -k_{y}^{2} \frac{m_{e}}{m_{i}},
\end{aligned}
$$

where $\omega_{d}=k_{y} \rho_{s} c_{s} / R_{1}, \quad \omega_{*}=k_{y} \rho_{s} c_{s} / L_{n}, \quad \omega_{\|}=k_{\|} c_{s}, \quad \eta=L_{n} / L_{T}$, $\nu=e^{2} n /\left(\sigma_{\|} m_{i}\right)$. The dispersion relation in Eq. (5) contains interchange and entropy modes in the $k_{\|}=0$ limit (i.e., in the $\omega_{\|}=0$ limit, denoted as interchange limit), ${ }^{27}$ and drift waves, made unstable by finite electron mass and resistivity effects, in the limit $R_{1} \rightarrow \infty$ (i.e., in the limit $\omega_{d}=0$, denoted as drift-wave limit). ${ }^{28}$ The mode described by the full dispersion relation, Eq. (5), is denoted as drift-interchange mode. Because of the magnetic geometry in the TORPEX device, the wavenumbers $k_{y}$ and $k_{\|}$have a discretized spectrum. The perturbations can be expressed as $\tilde{f}=\tilde{f} \exp \left(\operatorname{in} \varphi+i k_{z} z\right)$ $=\tilde{f} \exp \left(i k_{\varphi} R_{1} \varphi+i k_{z} z\right) \quad\left(\right.$ for $\quad B_{z} \ll B_{\varphi}, \quad z \simeq y$ and $k_{z} \simeq k_{y}$ ), $k_{z}=2 \pi l / L_{z}, L_{z}$ is the vertical distance between the bottom and the top of the vessel, at radius $R_{1}$, and $n, l=0, \pm 1, \pm 2, \ldots$. From $k_{\varphi}$ and $k_{z}$, the parallel wavenumber can be evaluated, $k_{\|}=\mathbf{k} \cdot \mathbf{B} / B=\left(n B_{\varphi} / R_{1}+k_{z} B_{z}\right) / \sqrt{B_{\varphi}^{2}+B_{z}^{2}}$. 

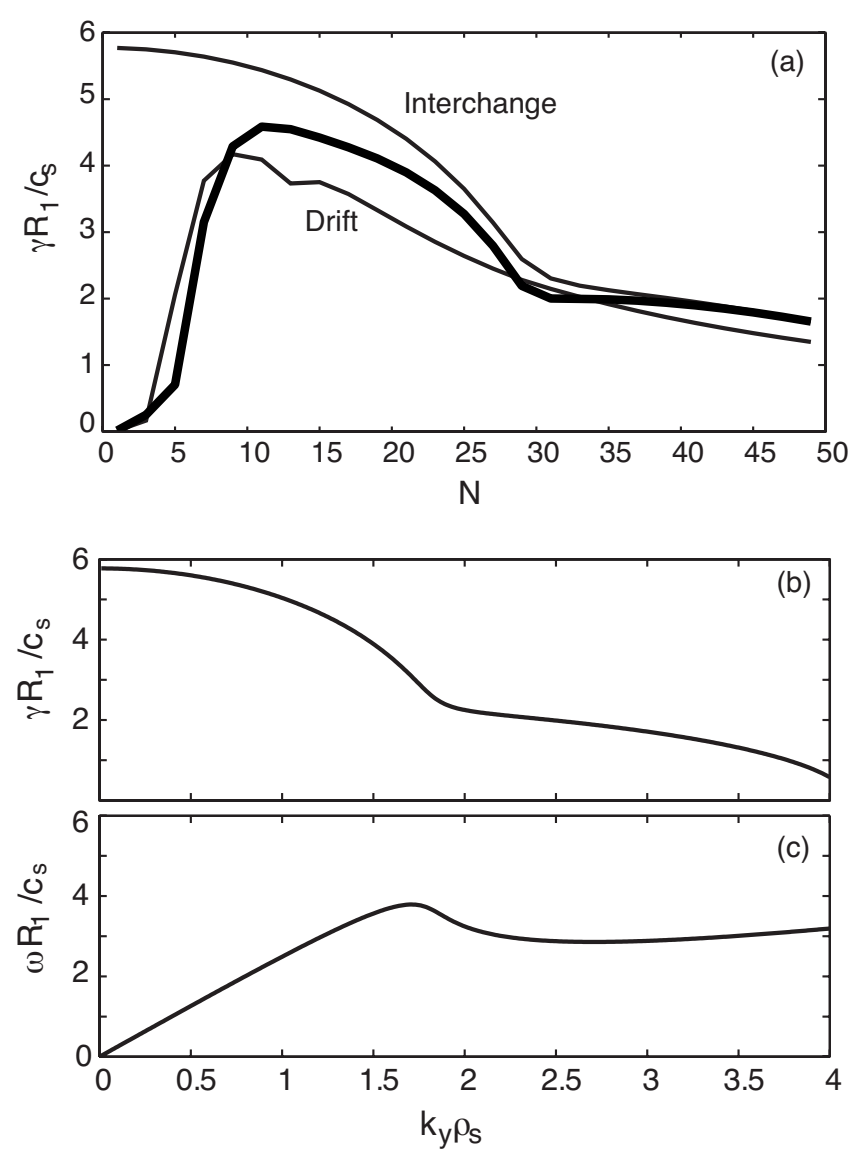

FIG. 11. (a) Dependence of the normalized growth rate of the driftinterchange (thick line) and of the drift and interchange instabilities on the vertical field. The value of the vertical field is parametrized by $N$, the number of toroidal turns of a magnetic field line, the larger $N$ the lower $B_{z}$. (b, c) Analytical solution of the dispersion relation in the limit of interchange instabilities.

Using the fact that $B_{z} \ll B_{\varphi}$, and writing $k_{\|}=2 \pi m / L_{c}$ ( $N$ is assumed to be an integer), one obtains $m=l+N n$.

Figure 11 shows the maximum growth rate of the instability predicted by Eq. (5) as a function of $N$, in the interchange limit, in the drift wave limit, and for the full driftinterchange dispersion relation (thick line). The modes with $k_{\|}=0$ are excluded in the latter case to isolate the contribution from the interchange instability. The maximum growth rate is evaluated over the allowed $k_{y}$ and $k_{\|}$. We use $\eta=1$, $L_{z}=100 \rho_{s}, R_{1} / L_{n}=10, \nu R_{1} / c_{s}=m_{e} R_{1} /\left(m_{i} c_{s} \tau_{e}\right)=0.001$, which correspond to the typical values measured in the experiments discussed herein. With increasing $N$ (i.e., decreasing $B_{z}$ ) the growth rate of the drift-interchange mode increases with respect to the interchange mode; their growth rates become comparable at $N \simeq 10$, which corresponds to $B_{z} \simeq 0.4 \mathrm{mT}$. With focus on the interchange mode (i.e., the mode with $m=0)$, its wavenumber is discretized according to $k_{z}=2 \pi n /\left(L_{z} / N\right)=2 \pi n / \Delta$. The growth rate and the frequency of the mode as a function of $k_{z}$ are shown in Figs. 11(b) and 11(c). The peak growth rate is localized at the lowest allowed $k_{z}, k_{z}=2 \pi / \Delta$. This value is in good agreement with the measured $k_{z}$, as shown in Fig. 9. The frequency shown in Figs. 11(b) and 11(c) is calculated in the plasma frame. In the laboratory frame the measured frequency is Doppler shifted by the $\mathbf{E} \times \mathbf{B}$ velocity, by the amount $k_{z} v_{E}$. The $\mathbf{E} \times \mathbf{B}$ flow is treated as a constant background by simply inducing a Doppler shift in the measured frequency and is therefore not included in the model. As shown in the figure, at its maximum growth rate, the frequency of the interchange mode is below $1 \mathrm{kHz}$; the corresponding phase velocity, calculated for $k_{z}=40 \mathrm{~m}^{-1}$, is below $160 \mathrm{~m} / \mathrm{s}$, much lower than the phase velocity of $1.8 \mathrm{~km} / \mathrm{s}$ induced by the $\mathbf{E} \times \mathbf{B}$ flow, in agreement with the measurements, Fig. 10(c).

\section{CONCLUSIONS}

Both drift and interchange instabilities are observed in TORPEX plasmas. They coexist in the unfavorable curvature region for a limited range of values of the vertical magnetic field. The drift mode is dominant for low values of $B_{z}$, corresponding to a long magnetic field connection length between top and bottom of the vacuum vessel. A gradual transition to a pure interchange instability is observed when the magnitude of the magnetic vertical field is increased. The observed transition is consistent with the predictions of a two-fluid linear model, based on the drift-reduced Braginskii equations, ${ }^{17}$ which takes into account the curvature of the magnetic field, resistivity, and finite electron mass effects (i.e., the drive for both the interchange and the drift instability). ${ }^{8}$ Consistent with the observations, the drift instability is found to dominate for the lowest values of the vertical field, while the interchange instability is dominating for large vertical fields.

The plasma conditions investigated on TORPEX exhibit a close similarity with those of tokamak SOL, characterized by a magnetic field configuration with open field lines. Plasma parameters and the high relative fluctuation levels, driven by strong, localized pressure gradients, are comparable. Similar results are also found in terms of statistical properties of fluctuations, which are dictated in TORPEX plasmas by the dynamics in the drift-interchange range of frequencies. ${ }^{29}$ Other phenomena are commonly observed in both tokamak SOL and TORPEX plasmas, such as the anomalously high level of turbulent cross-field transport associated with instabilities and with propagating macroscopic structures, or blobs. ${ }^{24}$ On TORPEX, drift-interchange instabilities are ultimately responsible for the generation of blobs. This effect is particularly evident at the largest values of the vertical magnetic field, for which the background profiles have a pronounced slab character and the instability has an interchange character. ${ }^{22,23}$ A similar relationship between instabilities and blobs is compatible with results obtained in tokamaks, ${ }^{30,31}$ as suggested by numerical simulations. ${ }^{32}$ Therefore, the study of cross-field transport would greatly benefit from a deeper understanding of the nature of the driftinterchange instabilities.

The results presented in this paper may have a direct impact for fusion plasmas, in that they provide a useful benchmark for the modeling of SOL turbulence. Drift and interchange modes can coexist in the region across the Last Close Flux Surface. Depending on the dominant character of the instabilities, different regimes, possibly with a different impact on the cross-field transport, could be achieved. The 
transition between regimes may be ascribed to a single parameter, such as the connection length for TORPEX. The identification of and the possibility of controlling such parameter may represent a considerable step forward for a better understanding of SOL physics.

\section{ACKNOWLEDGMENTS}

The authors acknowledge fruitful discussions with B. N. Rogers, I. Furno, B. Labit, and A. Diallo.

Ricci is supported by a EURATOM Fusion Fellowship. This work is supported in part by the Swiss National Science Foundation.

${ }^{1}$ N. A. Krall, Advances in Plasma Physics, edited by A. Simon and W. B. Thomson (Interscience, New York, 1968).

${ }^{2}$ W. Horton, Phys. Rep. 192, 1 (1990).

${ }^{3}$ R. J. Goldston and P. H. Rutherford, Introduction to Plasma Physics (Institute of Physics, New York, 1995), Chaps. 11-12.

${ }^{4}$ A. B. Mikhailovskii, Electromagnetic Instabilities in an Inhomogeneous Plasma (Institute of Physics, Philadelphia, 1992).

${ }^{5}$ O. Garcia, V. Naulin, A. Nielsen, and J. J. Rasmussen, Phys. Rev. Lett. 92, 165003 (2004)

${ }^{6}$ B. D. Scott, Phys. Plasmas 12, 062314 (2005).

${ }^{7}$ O. E. Garcia, J. Horacek, and R. A. Pitts, Plasma Phys. Controlled Fusion 48, L1 (2006).

${ }^{8}$ P. Ricci, B. N. Rogers, and S. Brunner, "High and low confinement modes in simple magnetized toroidal plasmas," Phys. Rev. Lett (submitted).

${ }^{9}$ A. Fasoli, B. Labit, M. M. Grath, S. H. Müller, G. Plyushchev, M. Podestà, and F. M. Poli, Phys. Plasmas 13, 055902 (2006).

${ }^{10}$ K. Rypdal and S. Ratynskaia, Phys. Rev. Lett. 94, 225002 (2005).

${ }^{11}$ F. M. Poli, S. Brunner, A. Diallo, A. Fasoli, I. Furno, B. Labit, S. H. Müller, G. Plyushchev, and M. Podestà, Phys. Plasmas 13, 102104 (2006).
${ }^{12}$ F. M. Poli, M. Podestà, and A. Fasoli, Phys. Plasmas 14, 052311 (2007).

${ }^{13}$ F. Brochard, E. Gravier, and G. Bonhomme, Phys. Plasmas 12, 062104 (2005).

${ }^{14}$ G. Prasad, D. Bora, Y. C. Saxena, and G. C. Sethia, Plasma Phys. Controlled Fusion 37, 387 (1995).

${ }^{15}$ F. Greiner, D. Block, and A. Piel, Contrib. Plasma Phys. 44, 335 (2004).

${ }^{16}$ J. C. Perez, W. Horton, K. G. W. L. Rowan, K. Lee, and R. B. Dahlburg, Phys. Plasmas 13, 032101 (2006).

${ }^{17}$ A. Zeiler, J. F. Drake, and B. Rogers, Phys. Plasmas 4, 2134 (1997).

${ }^{18}$ F. F. Chen and T. Sekiguchi, J. Appl. Phys. 36, 2363 (1965).

${ }^{19}$ M. Podestà, A. Fasoli, B. Labit, M. McGrath, S. H. Müller, and F. M. Poli, Plasma Phys. Controlled Fusion 47, 1989 (2005).

${ }^{20}$ F. M. Poli, Ph.D. thesis, EPFL, 2007, http://library.epfl.ch/theses/?nr $=3849$.

${ }^{21}$ D. Jassby, Phys. Fluids 15, 1590 (1972).

${ }^{22}$ I. Furno, B. Labit, M. Podestà, A. Fasoli, S. H. Müller, F. M. Poli, P. Ricci, C. Theiler, S. Brunner, A. Diallo, and J. Graves, Phys. Rev. Lett. 100, 055004 (2008).

${ }^{23}$ I. Furno, B. Labit, A. Fasoli et al., Phys. Plasmas 15, 055903 (2008).

${ }^{24}$ M. Podestà, Ph.D. thesis, EPFL, 2007, http://library.epfl.ch/theses/?nr $=3765$.

${ }^{25}$ J. M. Beall, Y. C. Kim, and E. J. Powers, J. Appl. Phys. 53, 3933 (1982).

${ }^{26}$ S. I. Braginskii, Rev. Plasma Phys. 1, 205 (1965).

${ }^{27}$ P. Ricci, B. N. Rogers, W. Dorland, and M. Barnes, Phys. Plasmas 13, 062102 (2006).

${ }^{28}$ B. Rogers and W. Dorland, Phys. Plasmas 12, 062511 (2005).

${ }^{29}$ B. Labit, I. Furno, A. Fasoli, A. Diallo, S. H. Müller, G. Plyushchev, M. Podestà, and F. M. Poli, Phys. Rev. Lett. 98, 255002 (2007).

${ }^{30}$ J. Terry, S. Zweben, K. Hallatschek, B. LaBombard, R. Maqueda, B. Bai, C. Boswell, M. Greenwald, D. Kopon, W. Nevins, et al., Phys. Plasmas 10, 1739 (2003)

${ }^{31}$ S. Zweben, R. Maqueda, D. Stotler, A. Keesee, J. Boedo, C. Bushand, S. Kaye, B. LeBlanc, J. Lowrance, V. Mastrocola, et al., Nucl. Fusion 44, 134 (2004).

${ }^{32}$ J. Myra, D. D'Ippolito, D. Stotler, S. Zweben, B. LeBlanc, J. Menard, R. Maqueda, and J. Boedo, Phys. Plasmas 13, 092509 (2006). 